This item was submitted to Loughborough's Research Repository by the author.

Items in Figshare are protected by copyright, with all rights reserved, unless otherwise indicated.

\title{
Electron relaxation in metals: theory and exact analytical solutions
}

PLEASE CITE THE PUBLISHED VERSION

PUBLISHER

(C) American Physical Society

VERSION

VoR (Version of Record)

LICENCE

CC BY-NC-ND 4.0

REPOSITORY RECORD

Kabanov, V.V., and A.S. Alexandrov. 2019. "Electron Relaxation in Metals: Theory and Exact Analytical Solutions". figshare. https://hdl.handle.net/2134/4275. 


\title{
Electron relaxation in metals: Theory and exact analytical solutions
}

\author{
V. V. Kabanov and A. S. Alexandrov \\ Josef Stefan Institute, 1001 Ljubljana, Slovenia \\ and Department of Physics, Loughborough University, Loughborough LE11 3TU, United Kingdom \\ (Received 4 September 2008; revised manuscript received 20 October 2008; published 13 November 2008)

\begin{abstract}
The nonequilibrium dynamics of electrons is of a great experimental and theoretical value, providing important microscopic parameters of the Coulomb and electron-phonon interactions in metals and other cold plasmas. Because of the mathematical complexity of collision integrals, theories of electron relaxation often rely on the assumption that electrons are in a "quasiequilibrium" (QE) with a time-dependent temperature, or on the numerical integration of the time-dependent Boltzmann equation. We transform the integral Boltzmann equation to a partial differential Schrödinger-type equation with imaginary time in a one-dimensional "coordinate" space reciprocal to energy which allows for exact analytical solutions in both cases of electron-electron and electron-phonon relaxations. The exact relaxation rates are compared with the QE relaxation rates at high and low temperatures.
\end{abstract}

DOI: 10.1103/PhysRevB.78.174514

PACS number(s): 71.38.-k, 74.40.+k, 72.15.Jf, 74.72.- $-\mathrm{h}$

\section{INTRODUCTION}

In recent years investigations of photoresponse functions in advanced materials have gone through a vigorous revival. In particular, laser "pump-probe" techniques, where a second probe pulse is delayed in time with respect to the pump pulse, provide unique information on the strength of electron-electron (e-e) and electron-phonon (e-ph) interactions in metals and doped insulators if an adequate theory is in place.

At present detailed experimental data on relaxation processes are collected for metals ${ }^{1-5}$ and high-temperature superconductors. ${ }^{6-12}$ The pump-probe experiments are routinely analyzed in the framework of the so-called twotemperature model (TTM). ${ }^{13,14}$ The model is based on the assumption that electrons and phonons are in a thermal quasiequilibrium $(\mathrm{QE})$ with two different time-dependent temperatures $T_{e}(t)$ and $T_{l}(t)$. The comprehensive analysis of experimental data collected at room temperature ${ }^{1}$ allowed for a determination of the e-ph coupling constant $\lambda$ of many metals and low-temperature superconductors in the framework of TTM.

Similar experiments and their analysis were performed on high-temperature superconductors. The femtosecond timeresolved measurements on the high- $T_{c}$ superconductors $\mathrm{Tl}_{2} \mathrm{Ba}_{2} \mathrm{Ca}_{2} \mathrm{Cu}_{3} \mathrm{O}_{10}$ (Ref. 6) and $\mathrm{YBa}_{2} \mathrm{Cu}_{3} \mathrm{O}_{7-\delta}$ (Refs. 7 and 8) found a relaxation process below $T_{c}$, which is distinct from the equilibration of hot carriers in the normal state. A relatively strong e-ph coupling, $\lambda \simeq 0.9,{ }^{8}$ and a rapid decrease in the photoresponse decay rate with decreasing temperature ${ }^{15}$ were found in $\mathrm{YBa}_{2} \mathrm{Cu}_{3} \mathrm{O}_{6.5}$, and the phonon bottleneck $^{12,16-18}$ or a biparticle recombination ${ }^{15,19}$ were observed below $T_{c}$. More recently, a time-resolved photoemission spectroscopy ${ }^{20}$ and the standard pump-probe optical measurements ${ }^{21}$ have been performed on $\mathrm{Bi}_{2} \mathrm{Sr}_{2} \mathrm{CaCu}_{2} \mathrm{O}_{8+\delta}$. Their TTM analysis has led to a rather weak e-ph coupling, $\lambda<0.25$.

The pump-probe techniques have a potential to resolve a controversial issue on whether the e-ph interaction is crucial $^{22}$ or weak and inessential ${ }^{23}$ for the mechanism of high-temperature superconductivity. The pioneering work by Kaganov et al. ${ }^{13}$ and subsequent TTM studies were based on the assumption that electrons are in the thermal QE state because the e-e relaxation time is supposed to be much shorter than the e-ph relaxation time. This assumption is of course incorrect on a femtosecond scale comparable with the e-e scattering time of highly excited electrons. But the expectation has been that deviation from QE may not in fact have much influence on the electron energy relaxation on a larger time scale ${ }^{14}$ (for discussions of TTM with respect to some experiments, see, for example, Refs. 24 and 25).

However, later on it has been realized that nonthermal effects are essential even on the picosecond scale, comparable with the e-ph relaxation time, when conditions of low laser excitation power and relatively low temperature are chosen. ${ }^{26}$ Under these conditions, the e-e collision rate becomes strongly suppressed as a result of the Pauli exclusion principle. Numerically integrating the Boltzmann equation with e-e and e-ph collision integrals, Groeneveld et al. ${ }^{26}$ showed that the electron gas cannot attain a thermal distribution by e-e collisions on the time scale of the e-ph energy relaxation. A departure from $\mathrm{QE}$ leads to an increase in the e-ph energy relaxation time with respect to the QE expectation. As a consequence of this departure, one might underestimate the e-ph coupling using TTM.

While numerical integrations of the Boltzmann equation can describe the time evolution of the electron distribution function on any time scale, they require a number of input parameters, which might be unknown a priori. Here, an analytical approach to this long-standing problem is developed. We reduce the integral Boltzmann equation to a differential Schrödinger-type equation using an auxiliary space reciprocal to energy and find exact analytical time-dependent distributions of electrons in both cases of electron-electron and electron-phonon relaxations. We also derive long-time relaxation rates of response functions and compare them with TTM.

\section{ELECTRON-ELECTRON RELAXATION}

Let us first consider a nonthermal relaxation of the electron distribution function $f_{\mathbf{k}}(t)$ caused by electron-electron 
collisions, which is described by the following Boltzmann equation:

$$
\begin{aligned}
\dot{f}_{\mathbf{k}}= & \frac{2 \pi}{\hbar} \sum_{\mathbf{p}, \mathbf{q}} V_{c}^{2}(\mathbf{q}) \delta\left(\xi_{\mathbf{k}}+\xi_{\mathbf{p}}-\xi_{\mathbf{k}+\mathbf{q}}-\xi_{\mathbf{p}-\mathbf{q}}\right) \\
& \times\left[f_{\mathbf{k}+\mathbf{q}} f_{\mathbf{p}-\mathbf{q}}\left(1-f_{\mathbf{k}}\right)\left(1-f_{\mathbf{p}}\right)-f_{\mathbf{k}} f_{\mathbf{p}}\left(1-f_{\mathbf{k}+\mathbf{q}}\right)\left(1-f_{\mathbf{p}-\mathbf{q}}\right)\right] .
\end{aligned}
$$

Here $\dot{f}_{\mathbf{k}} \equiv \partial f_{\mathbf{k}}(t) / \partial t, \quad V_{c}(\mathbf{q})$ is the matrix element of the electron-electron scattering (pseudo)potential, and $\xi_{\mathbf{k}}$ is the electron energy with respect to the equilibrium chemical potential. For transparency we drop the time argument in the distribution function. If the distribution function depends only on energy and time, $f_{\mathbf{k}}=f_{\xi}$, one can average this equation over the angles of $\mathbf{k}$ as

$$
\dot{f}_{\xi} \equiv N^{-1}(\xi) \sum_{\mathbf{k}} \delta\left(\xi_{\mathbf{k}}-\xi\right) \dot{f}_{\mathbf{k}}
$$

where $N(\xi)$ is the density of states (DOS) per spin, with the following result:

$$
\begin{aligned}
\dot{f}_{\xi}= & \iiint d \xi^{\prime} d \epsilon d \epsilon^{\prime} K\left(\xi, \xi^{\prime}, \epsilon, \epsilon^{\prime}\right) \delta\left(\xi+\epsilon-\xi^{\prime}-\epsilon^{\prime}\right) \\
& \times\left[f_{\xi^{\prime}} f_{\epsilon^{\prime}}\left(1-f_{\xi}\right)\left(1-f_{\epsilon}\right)-f_{\xi} f_{\epsilon}\left(1-f_{\xi^{\prime}}\right)\left(1-f_{\epsilon^{\prime}}\right)\right]
\end{aligned}
$$

where

$$
\begin{aligned}
K\left(\xi, \xi^{\prime}, \boldsymbol{\epsilon}, \boldsymbol{\epsilon}^{\prime}\right)= & \frac{2 \pi}{\hbar N(\xi)} \sum_{\mathbf{k}, \mathbf{p}, \mathbf{q}} V_{c}^{2}(\mathbf{q}) \delta\left(\xi_{\mathbf{k}}-\xi\right) \delta\left(\xi_{\mathbf{p}}-\epsilon\right) \\
& \times \delta\left(\xi_{\mathbf{k}+\mathbf{q}}-\xi^{\prime}\right) \delta\left(\xi_{\mathbf{p}-\mathbf{q}}-\epsilon^{\prime}\right) .
\end{aligned}
$$

We restrict our theory to relaxations involving nonequilibrium electron-hole excitations with energies much less than the equilibrium Fermi energy $E_{F}$. Since the kernel $K\left(\xi, \xi^{\prime}, \epsilon, \epsilon^{\prime}\right)$ has variation on the scale of the Fermi energy, one can approximate it by a constant, $K\left(\xi, \xi^{\prime}, \epsilon, \epsilon^{\prime}\right) \approx K$. This constant is related to the Coulomb pseudopotential $\mu_{C}$ $\equiv V_{c} N(0)$, important in the theory of superconductivity, $K$ $\approx \pi \mu_{C}^{2} / 2 \hbar E_{F}$. Assuming a low laser excitation power, we linearize Eq. (3) by introducing a small nonequilibrium correction, $\phi(\xi, t) \ll 1$, to the equilibrium distribution $n_{\xi}$,

$$
f_{\xi}=n_{\xi}+\phi(\xi, t)
$$

where $n_{\xi}=\left(e^{\xi / k_{B} T}+1\right)^{-1}$. Keeping terms linear in $\phi(\xi, t)$ and measuring energies in units of $k_{B} T$, which is the only relevant energy scale of the problem, one obtains

$$
\begin{aligned}
\dot{\phi}(\xi, t)= & K\left(k_{B} T\right)^{2} \iiint d \xi^{\prime} d \epsilon d \epsilon^{\prime} \delta\left(\xi+\epsilon-\xi^{\prime}-\epsilon^{\prime}\right) \\
& \times\left[\phi\left(\xi^{\prime}, t\right)\left(n_{-\xi^{\prime}} n_{-\epsilon} n_{\epsilon^{\prime}}+n_{\xi} n_{\epsilon^{\prime}} n_{-\epsilon^{\prime}}\right)\right. \\
& \left.-\phi(\xi, t)\left(n_{\xi^{\prime}} n_{-\epsilon} n_{\epsilon^{\prime}}+n_{-\xi^{\prime}} n_{\epsilon^{\prime}} n_{-\epsilon^{\prime}}\right)\right] .
\end{aligned}
$$

Performing simple integrations in linearized Eq. (6) yields

$$
\begin{aligned}
\dot{\phi}(\xi, t)= & -\frac{\phi(\xi, t)}{\tau_{\mathrm{e}}(\xi)}+\frac{K\left(k_{B} T\right)^{2}}{\cosh (\xi / 2)} \int_{-\infty}^{\infty} d \xi^{\prime} \phi\left(\xi^{\prime}, t\right) \cosh \left(\xi^{\prime} / 2\right) \\
& \times\left[\frac{\xi-\xi^{\prime}}{\sinh \left(\frac{\xi-\xi^{\prime}}{2}\right)}-\frac{\xi+\xi^{\prime}}{2 \sinh \left(\frac{\xi+\xi^{\prime}}{2}\right)}\right],
\end{aligned}
$$

where

$$
\tau_{\mathrm{e}}(\xi)=\frac{2}{\left(\pi^{2}+\xi^{2}\right) K\left(k_{B} T\right)^{2}}
$$

is the familiar lifetime of electron-hole excitations in the Fermi liquid. Here we have used the integral $\int_{0}^{\infty} d z \ln (z) /[(z$ $-a)(z+b)]=\left[\pi^{2}-\ln ^{2}(a)+\ln ^{2}(b)\right] / 2(a+b)$, with $a, b>0$.

The second term on the right-hand side of Eq. (7) describes a source of quasiparticles due to inelastic electronelectron collisions. Collisions in cold degenerate plasmas differ essentially from quasielastic collisions in classical (hot) plasmas. In the latter the energy transfer is small compared with the electron energy due to the long-range character of the Coulomb potential, so that one can approximate the Boltzmann collision integral by the differential LandauFokker-Planck (LFP) equation (see, for example, Ref. 27). As one can see from Eq. (7), it is not the case in metals. The collision energy transfer in metals is about the same as the excitation energy itself, which makes the differential LFP approximation unacceptable here.

Remarkably, the electron-electron collision integral acquires a differential form in a reciprocal auxiliary-time space introduced via the Fourier transform of Eq. (7), rather than in the energy space as in the LFP case. Let us consider nonequilibrium states conserving the electron-hole symmetry, so that the nonequilibrium part of the distribution is an odd function of energy, $\phi(-\xi, t)=-\phi(\xi, t)$. If one determines a function

$$
\chi(\xi, t) \equiv \phi(\xi, t) \cosh (\xi / 2),
$$

then the Boltzmann equation is simplified as

$$
\frac{\dot{\chi}(\xi, t)}{K\left(k_{B} T\right)^{2}}=-\frac{\pi^{2}+\xi^{2}}{2} \chi(\xi, t)+\frac{3}{2} \int_{-\infty}^{\infty} d \xi^{\prime} \chi\left(\xi^{\prime}, t\right) \frac{\xi-\xi^{\prime}}{\sinh \left(\frac{\xi-\xi^{\prime}}{2}\right)} .
$$

We shall see below that a "bound state" of the effective "Schrödinger" equation for the Fourier transform of $\chi(\xi, t)$ corresponds to the stationary quasiequilibrium distribution. Taking the Fourier transform of Eq. (10), we arrive at an exact differential counterpart of the Boltzmann equation,

$$
\tau_{\mathrm{e}} \dot{\psi}(x, t)=\left[\frac{\partial^{2}}{\partial x^{2}}+\frac{6}{\cosh ^{2}(x)}-1\right] \psi(x, t),
$$

where

$$
\tau_{\mathrm{e}}=2 / \pi^{2} K\left(k_{B} T\right)^{2}
$$

and 


$$
\psi(x, t)=\int_{-\infty}^{\infty} d \xi \chi(\xi, t) e^{i x \xi / \pi} .
$$

Here, another integral $\int_{-\infty}^{\infty} d z z \exp (i x z) / \sinh (z / 2)=2 \pi^{2}$ / $\cosh ^{2}(\pi x)$ has been used.

The solution of Eq. (11) is found as a superposition of normalized eigenstates, $\psi_{k}(x)$, of a textbook Hamiltonian, ${ }^{28}$

$$
\psi(x, t)=\sum_{k} c_{k} \psi_{k}(x) e^{\left(k^{2}-1\right) t / \tau_{\mathrm{e}}},
$$

where coefficients $c_{k}$ are determined by the initial nonequilibrium distribution function $\phi(\xi, 0)$ at $t=0$,

$$
c_{k}=\int_{-\infty}^{\infty} d x \int_{-\infty}^{\infty} d \xi \psi_{k}^{*}(x) \cosh (\xi / 2) \phi(\xi, 0) e^{i x \xi / \pi} .
$$

The eigenstates $\psi_{k}(x)$ and the eigenvalues $E=-k^{2}$ are found from the Schrödinger equation

$$
\left[\frac{\partial^{2}}{\partial x^{2}}+\frac{m(m+1)}{\cosh ^{2}(x)}\right] \psi_{k}(x)=k^{2} \psi_{k}(x),
$$

with $m=2$. This equation has a finite number of discrete bound states with real $k$ 's (Ref. 28) and continuum extended states with imaginary $k$ 's, $k=i p$ ( $p$ is real). If $m$ is an integer, there are $m$ bound states with $k=1,2, \ldots, m$ and both bound and unbound eigenstates can be expressed in terms of elementary functions, ${ }^{29}$

$$
\psi_{k}(x)=A_{k} \hat{D}_{m} \hat{D}_{m-1} \cdots \hat{D}_{1} e^{k x},
$$

where $A_{k}$ is the normalizing amplitude and $\hat{D}_{m}=d / d x$ $-m \tanh (x)$. In our case $(m=2)$ there are two bound states, the even ground state with $k=2(E=-4)$ and the odd excited state with $k=1 \quad(E=-1)$. For relaxations conserving the electron-hole symmetry, the ground-state contribution to the superposition, Eq. (14), is integrated to zero because the initial nonequilibrium distribution is odd. On the contrary, the excited odd state with $\psi_{1}(x) \propto \sinh (x) / \cosh ^{2}(x)$ is the only state which survives in Eq. (14) at $t \rightarrow \infty$, so that

$$
\psi(x, \infty) \propto \frac{\sinh (x)}{\cosh ^{2}(x)},
$$

and [using Eqs. (9) and (13)]

$$
\phi(x, \infty) \propto \frac{\xi}{\cosh ^{2}(\xi / 2)},
$$

which is precisely the result of the $\mathrm{QE}$ approximation. Indeed expanding the $\mathrm{QE}$ distribution function $f_{\mathrm{QE}}$ $=\left\{\exp \left[\left(E-E_{F}\right) / T_{e}\right]+1\right\}^{-1}$ in powers of $T_{e}-T$, we obtain

$$
f_{\mathrm{QE}}=n_{\xi}+\left(\frac{T_{e}-T}{4 T}\right) \frac{\xi}{\cosh ^{2}(\xi / 2)},
$$

with the same nonequilibrium correction $n_{\mathrm{QE}} \propto \xi / \cosh ^{2}(\xi / 2)$, as in Eq. (19).

The exact solution, Eq. (14), allows us to trace the relaxation at any energy and at any time scale. In particular, Fig. 1 represents the time evolution of the total number of nonequilibrium excitations (electrons plus holes), $n$

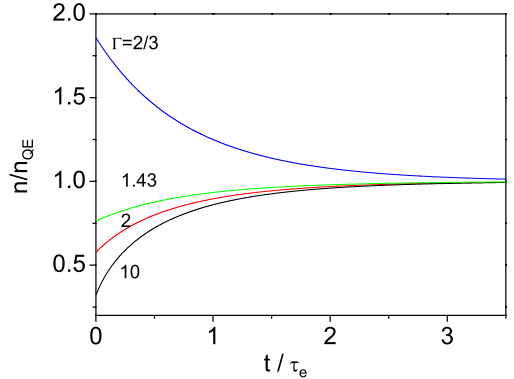

FIG. 1. (Color online) Relaxation of the total number of nonequilibrium excitations, $n$, normalized by the quasiequilibrium number $n_{\mathrm{QE}}$ for different widths $\Gamma$ of the initial nonequilibrium distribution.

$=2 \int_{0}^{\infty} d \xi \phi(\xi, t)$, for initial nonequilibrium distributions of the shape, $\phi(\xi, 0)=\left(\pi^{1 / 2} E_{0} / 2 \Gamma^{3}\right) \xi \exp \left[-(\xi / \Gamma)^{2}\right]$, where the distribution width $\Gamma$ is varied but the total energy $E_{0}$ is unchanged. For wide sources with large $\Gamma>1$, the number of excitations increases with time, conserving the total energy in the process of their cooling. On the other hand, when most excitations at $t=0$ are created with the energy less than $k_{B} T$ (i.e., $\Gamma<1$ ), their number decreases with time since their individual energies increase due to collisions with equilibrium electrons. One can trace the relaxation for any initial distribution including the case where a single photon initially creates a single electron-hole pair, which cascades eventually into a large number of pairs until they get lost in the background thermal distribution.

The asymptotic behavior of response functions can be readily obtained from Eq. (14) by taking into account that only the excited bound state and the extended states with small $p \approx\left(t / \tau_{e}\right)^{-1 / 2} \ll 1$ contribute to the sum in Eq. (14), when $t / \tau_{\mathrm{e}} \gg\left(1,1 / \Gamma^{2}\right)$. Substituting the extended eigenfunctions $\psi_{p}(x) \propto\left[3 \tanh ^{2}(x)-3 i p \tanh (x)-p^{2}-1\right] \exp ($ ipx $)$ into Eq. (14) with $c_{p} \propto p$ at small $p$ and integrating over $p$ yield

$$
\psi(x, t)-\psi(x, \infty) \propto \frac{x}{t^{3 / 2}} \exp \left(-\frac{t}{\tau_{e}}-\frac{x^{2} \tau_{\mathrm{e}}}{4 t}\right)
$$

in the saddle-point approximation. Performing the Fourier transform of Eq. (21) with respect to $x$, we find

$$
\phi(\xi, t)-\phi(\xi, \infty) \propto \xi e^{-t / \tau_{\mathrm{e}}(\xi)} .
$$

The same result is obtained by using a $\tau$ approximation for Boltzmann equation (7),

$$
\dot{\phi}(\xi, t)=-\frac{\phi(\xi, t)-\phi(\xi, \infty)}{\tau_{e}(\xi)},
$$

which has the following solution:

$$
\phi(\xi, t)=\phi(\xi, \infty)+[\phi(\xi, 0)-\phi(\xi, \infty)] e^{-t / \tau_{\mathrm{e}}(\xi)} .
$$

Hence one can use the $\tau$ approximation, Eq. (24), on the time scale much longer than the characteristic collision time $\tau_{\mathrm{e}}$. However this approximation is inaccurate on a shorter time scale because in contrast with the exact solution, Eq. (14), it does not conserve the total energy.

Integrating Eq. (22) yields a universal time asymptotic of the total number of electron-hole excitations, 


$$
n(t)-n_{\mathrm{QE}} \propto \frac{e^{-t / \tau_{\mathrm{e}}}}{t},
$$

as also seen from Fig. 1.

Importantly the characteristic e-e relaxation time is quite long due to the Pauli exclusion principle. Using realistic $\mu_{C}=1$ and $E_{F}=10 \mathrm{eV}$, we estimate $\tau_{\mathrm{e}} \approx 1.2 \mathrm{ps}$ at the room temperature $T=300 \mathrm{~K}$ (see also Ref. 26), which increases further as $1 / T^{2}$ with cooling.

\section{ELECTRON-PHONON RELAXATION}

Now let us consider the electron-phonon relaxation described by the e-ph collision integral,

$$
\begin{aligned}
\dot{f}_{\mathbf{k}}= & \frac{2 \pi}{\hbar} \sum_{\mathbf{q}} M^{2}(\mathbf{q})\left\{\left[f_{\mathbf{k}-\mathbf{q}}\left(1-f_{\mathbf{k}}\right) N_{\mathbf{q}}-f_{\mathbf{k}}\left(1-f_{\mathbf{k}-\mathbf{q}}\right)\left(N_{\mathbf{q}}+1\right)\right]\right. \\
& \times \delta\left(\xi_{\mathbf{k}}-\xi_{\mathbf{k}-\mathbf{q}}-\hbar \omega_{\mathbf{q}}\right)+\left[f_{\mathbf{k}+\mathbf{q}}\left(1-f_{\mathbf{k}}\right)\left(N_{\mathbf{q}}+1\right)\right. \\
& \left.\left.-f_{\mathbf{k}}\left(1-f_{\mathbf{k}+\mathbf{q}}\right) N_{\mathbf{q}}\right] \delta\left(\xi_{\mathbf{k}}-\xi_{\mathbf{k}+\mathbf{q}}+\hbar \omega_{\mathbf{q}}\right)\right\},
\end{aligned}
$$

where $M(\mathbf{q})$ is the matrix element of the deformation potential and $N_{\mathbf{q}}$ is the distribution function of phonons with the frequency $\omega_{\mathbf{q}}$.

As in the former case of the e-e collisions, we average this equation over the momentum angles using Eq. (2) and conventional units:

$$
\begin{aligned}
\dot{f}_{\xi}= & 2 \pi \int d \omega \int d \xi^{\prime} Q\left(\omega, \xi, \xi^{\prime}\right)\left\{\delta\left(\xi-\xi^{\prime}-\hbar \omega\right)\right. \\
& \times\left[\left(f_{\xi^{\prime}}-f_{\xi}\right) N_{\omega}-f_{\xi}\left(1-f_{\xi^{\prime}}\right)\right]+\delta\left(\xi-\xi^{\prime}+\hbar \omega\right) \\
& \left.\times\left[\left(f_{\xi^{\prime}}-f_{\xi}\right) N_{\omega}+f_{\xi^{\prime}}\left(1-f_{\xi}\right)\right]\right\} .
\end{aligned}
$$

Here

$$
Q\left(\omega, \xi, \xi^{\prime}\right)=\frac{1}{\hbar N(\xi)} \sum_{\mathbf{k}, \mathbf{q}} M^{2}(\mathbf{q}) \delta\left(\xi_{\mathbf{k}-\mathbf{q}}-\xi^{\prime}\right) \delta\left(\xi_{\mathbf{k}}-\xi\right) \delta\left(\omega_{\mathbf{q}}-\omega\right)
$$

is the e-ph spectral function, ${ }^{14}$ which has $\omega$ variation on the scale of the maximum phonon frequency $\omega_{D}$ but has $\xi$ and $\xi^{\prime}$ variation only on a much larger energy scale on the order of $E_{F}$.

Linearizing Eq. (27) with the help of Eq. (5) yields

$$
\begin{aligned}
\dot{\phi}(\xi, t)= & 2 \pi \int d \omega \int d \xi^{\prime} Q\left(\omega, \xi, \xi^{\prime}\right)\left\{\left[\phi\left(\xi^{\prime}, t\right)\left(N_{\omega}+n_{\xi}\right)\right.\right. \\
& \left.-\phi(\xi, t)\left(N_{\omega}+n_{-\xi^{\prime}}\right)\right] \delta\left(\xi-\xi^{\prime}-\hbar \omega\right) \\
& +\left[\phi\left(\xi^{\prime}, t\right)\left(N_{\omega}+n_{-\xi}\right)-\phi(\xi, t)\left(N_{\omega}+n_{\xi^{\prime}}\right)\right] \\
& \left.\times \delta\left(\xi-\xi^{\prime}+\hbar \omega\right)\right\} .
\end{aligned}
$$

Characteristic electron energies in Eq. (29) are much less than the Fermi energy, so that

$$
Q\left(\omega, \xi, \xi^{\prime}\right) \approx Q(\omega, 0,0) \equiv \alpha^{2} F(\omega)
$$

is the familiar Eliashberg function. We also assume that phonons are in the thermal equilibrium, $N_{\omega}=\left[\exp \left(\hbar \omega / k_{B} T\right)\right.$
$-1]^{-1}$, due to their fast thermalization caused by anharmonic interactions (i.e., phonon-phonon collisions) and/or due to a small size of the sample and the pump-laser spot allowing for a fast escape of nonequilibrium phonons. If this condition is not satisfied, one has to solve an equation for the nonequilibrium phonon distribution coupled with Eq. (29), which is outside the scope of this paper. Under these assumptions Eq. (29) is transformed into a form similar to the e-e collision integral in Eq. (7),

$$
\begin{aligned}
\dot{\phi}(\xi, t)= & -\frac{\phi(\xi, t)}{\tau_{\mathrm{ph}}(\xi)}+\frac{2 \pi k_{B} T}{\hbar \cosh (\xi / 2)} \int_{-\infty}^{\infty} d \xi^{\prime} \\
& \times \operatorname{sgn}\left(\xi-\xi^{\prime}\right) \alpha^{2} F\left(\frac{k_{B} T\left|\xi-\xi^{\prime}\right|}{\hbar}\right) \\
& \times \frac{\cosh \left(\xi^{\prime} / 2\right)}{2 \sinh \left(\frac{\xi-\xi^{\prime}}{2}\right)} \phi\left(\xi^{\prime}, t\right),
\end{aligned}
$$

where

$$
\begin{aligned}
\frac{1}{\tau_{\mathrm{ph}}(\xi)}= & \frac{2 \pi k_{B} T}{\hbar} \int_{0}^{\infty} d \omega \alpha^{2} F\left(\frac{k_{B} T \omega}{\hbar}\right) \\
& \times\left[\frac{1}{\sinh \left(\frac{\omega}{2}\right) \cosh \left(\frac{\omega}{2}\right)}\right. \\
& \left.+\frac{\sinh ^{2}\left(\frac{\xi}{2}\right) \tanh \left(\frac{\omega}{2}\right)}{\cosh \left(\frac{\omega+\xi}{2}\right) \cosh \left(\frac{\omega-\xi}{2}\right)}\right]
\end{aligned}
$$

and energies are now measured in units of $k_{B} T$. The Eliashberg function is quite complicated in real metallic compounds because of their complex lattice structures. This complexity can be avoided in the high-temperature regime, $k_{B} T \gg \hbar \omega_{D}$, and in the opposite low-temperature regime, $k_{B} T \ll \hbar \omega_{D}$.

\section{A. High-temperature electron-phonon relaxation}

As shown by Allen ${ }^{14}$ the energy relaxation in TTM has a particularly simple form in terms of the moments of $\alpha^{2} F(\omega)$,

$$
\lambda\left\langle\omega^{n}\right\rangle \equiv 2 \int_{0}^{\infty} d \omega \frac{\alpha^{2} F(\omega) \omega^{n}}{\omega},
$$

where the coupling constant $\lambda$, which determines the critical temperature in the BCS superconductors, is

$$
\lambda=2 \int_{0}^{\infty} d \omega \frac{\alpha^{2} F(\omega)}{\omega} .
$$

Here we derive a high-temperature LFP-type equation for the nonequilibrium part of the distribution function $\phi(\xi, t)$ to compare our exact approach with the TTM results. ${ }^{13,14}$

At high temperatures the Eliashberg function is a narrow function on the temperature scale, so that one can apply a 
quasielastic approximation expanding the e-ph collision integral, Eq. (29) or (31), in powers of the phonon energy, $\hbar \omega$ $\ll k_{B} T$. The zero-order elastic terms are canceled out because the distribution function depends on energy only, while the next-order terms yield the LFP-type differential equation

$$
\gamma^{-1} \dot{\phi}(\xi, t)=\frac{\partial}{\partial \xi}\left[\tanh \left(\frac{\xi}{2}\right) \phi(\xi, t)+\frac{\partial}{\partial \xi} \phi(\xi, t)\right],
$$

where $\gamma=\pi \hbar \lambda\left\langle\omega^{2}\right\rangle / k_{B} T$. Apart from a numerical coefficient on the order of 1 , the characteristic e-ph relaxation rate $\gamma / 2$ is about the same as the TTM energy relaxation rate, ${ }^{14} \gamma_{T}$ $=3 \hbar \lambda\left\langle\omega^{2}\right\rangle / \pi k_{B} T$, at high temperatures. Indeed multiplying Eq. (35) by $\xi$ and integrating over all energies yield the rate of excitation energy relaxation,

$$
\dot{E}_{e}(t)=-\gamma \int_{-\infty}^{\infty} d \xi \tanh (\xi / 2) \phi(\xi, t),
$$

where $E_{e}(t)=\int_{-\infty}^{\infty} d \xi \xi \phi(\xi)$. If we replace $\tanh (\xi / 2)$ in this equation with its argument assuming that $\phi(\xi, t)$ has its characteristic energy width on the order of 1 , then $\dot{E}_{e}(t)$ $\approx-(\gamma / 2) E_{e}$. Hence the excitation energy relaxes as $E_{e}(t)$ $\propto \exp (-\gamma t / 2)$ almost independently of the particular shape of the nonequilibrium distribution.

To verify the numerical coefficient, one can substitute the TTM distribution $\phi_{\mathrm{TTM}}(\xi, t)=\left\{\left[T_{e}(t)-T\right] / 4 T\right\} \xi / \cosh ^{2}(\xi / 2)$ [Eq. (20)] into Eq. (36) to convert this into the temperature relaxation rate:

$$
\dot{T}_{e}(t)=-\frac{1}{2} \gamma\left(T_{e}-T\right) \frac{\int_{0}^{\infty} d x x \tanh (x) / \cosh ^{2}(x)}{\int_{0}^{\infty} d x x^{2} / \cosh ^{2}(x)} .
$$

Equation (37) is precisely the same as the TTM temperature rate, ${ }^{14} \dot{T}_{e}(t)=-\gamma_{T}\left(T_{e}-T\right)$, since the ratio of two integrals in Eq. (37) is $6 / \pi^{2}$.

According to Eq. (36) deviation of $\phi(\xi, t)$ from quasiequilibrium population does not have much influence on the energy relaxation, apart from a numerical coefficient. Hence TTM (Refs. 13 and 14) is the adequate approximation at high temperatures, which agrees well with experimental observations in conventional metals where Debye temperatures are rather low. ${ }^{1}$

\section{B. Low-temperature electron-phonon relaxation in poor metals}

Characteristic phonon frequencies are exceptionally high in many advanced materials such as copper oxides, $\hbar \omega_{D} / k_{B} \gtrsim 400-1000 \mathrm{~K}$, so that the low-temperature regime $k_{B} T \ll \hbar \omega_{D}$ is of great importance. Since all dimensionless energies in Eq. (31) are on the order of unity, one can apply a low-frequency asymptotic of $\alpha^{2} F(\omega)=\lambda n\left(\omega / \omega_{D}\right)^{n} / 2$ in this regime. The exponent $n$ depends on impurities, disorder, and sample dimensions: $n=2$ in clean bulk crystals, while $n=1$ in disordered metals due to a phonon damping ${ }^{30,31}$ and in metallic films. ${ }^{32}$ Then Eq. (31) can be Fourier transformed into the Schrödinger equation using Fourier transform equation (13) of $\chi(\xi, t) \equiv \phi(\xi, t) \cosh (\xi / 2)$.
In the poor-metal case $n=1$, the equation for $\chi(\xi, t)$ is almost the same as in the e-e case, Eq. (10), apart from a numerical coefficient in front of the integral term,

$$
\begin{aligned}
\frac{\dot{\chi}(\xi, t)}{\pi \lambda\left(k_{B} T\right)^{2} / \hbar^{2} \omega_{D}}= & -\frac{\pi^{2}+\xi^{2}}{2} \chi(\xi, t)+\frac{1}{2} \int_{-\infty}^{\infty} d \xi^{\prime} \chi\left(\xi^{\prime}, t\right) \\
& \times \frac{\xi-\xi^{\prime}}{\sinh \left(\frac{\xi-\xi^{\prime}}{2}\right)},
\end{aligned}
$$

where the integrals $\int_{0}^{\infty} d \omega \omega / \sinh (\omega / 2) \cosh (\omega / 2)=\pi^{2} / 2$ and $\int_{0}^{\infty} d \omega \omega \tanh (\omega / 2) / \cosh [(\omega+\xi) / 2] \cosh [(\omega-\xi) / 2]=\xi^{2} /$ $2 \sinh ^{2}(\xi / 2)$ have been used. The difference in the numerical coefficients in front of the integral terms originates in different statistics of scatterers, which are bosons in the e-ph case and fermions in the e-e case. At low temperatures the e-ph relaxation time has the same energy and temperature dependence as the e-e relaxation time [Eq. (8)],

$$
\tau_{\mathrm{ph}}(\xi)=\frac{2 \hbar^{2} \omega_{D}}{\left(\pi^{2}+\xi^{2}\right) \pi \lambda\left(k_{B} T\right)^{2}} .
$$

We also notice that the temperature dependence of the e-ph relaxation rate at low temperatures, $1 / \tau_{\mathrm{e}-\mathrm{ph}} \propto T^{2}$, is qualitatively different from its temperature dependence at high temperatures, $\gamma \propto 1 / T$.

The Fourier transform of Eq. (38) yields the Schrödingertype equation

$$
\tau_{\mathrm{ph}} \dot{\psi}(x, t)=\left[\frac{\partial^{2}}{\partial x^{2}}+\frac{2}{\cosh ^{2}(x)}-1\right] \psi(x, t),
$$

where

$$
\tau_{\mathrm{ph}}=\frac{2 \hbar^{2} \omega_{D}}{\pi^{3} \lambda\left(k_{B} T\right)^{2}} .
$$

Different from the e-e case [Eq. (16) with $m=2$ ], the steadystate Schrödinger equation

$$
\left[\frac{\partial^{2}}{\partial x^{2}}+\frac{2}{\cosh ^{2}(x)}\right] \psi_{k}(x)=k^{2} \psi_{k}(x)
$$

has only one bound (ground) state, $k=1$, and itinerant states with $k=i p$,

$$
\psi_{k}(x)=A_{k}[k-\tanh (x)] e^{k x}
$$

in the e-ph case $(m=1)$. Only itinerant states contribute to superposition equation (14) and determine the time relaxation of the distribution function because the contribution of the even ground state is integrated to zero and there is no excited odd state here. As a result, the nonequilibrium part of the distribution function and the number of excitations relax with characteristic time $\tau_{\mathrm{ph}}$ to zero rather than to any quasiequilibrium state as shown in Figs. 2 and 3 by lower curves $(\alpha=2)$. Their time asymptotic is found using the saddle-point approximation as in the case of the e-e collisions,

$$
\phi(\xi, t) \propto \xi e^{-t / \tau_{\mathrm{ph}}(\xi)},
$$




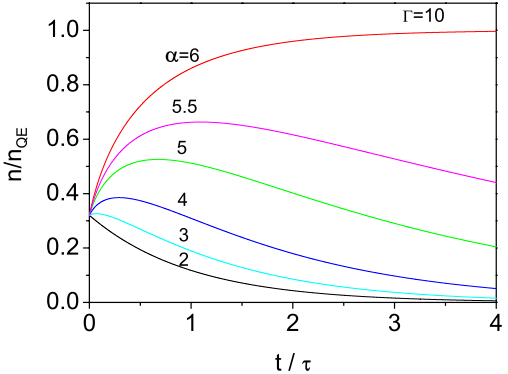

FIG. 2. (Color online) Relaxation of the total number of nonequilibrium excitations, $n$, for different e-e and e-ph scattering times characterized by parameter $\alpha$ [Eq. (51)] for the width $\Gamma=10$ of the initial nonequilibrium distribution.

$$
n(t) \propto \frac{e^{-t / \tau_{\mathrm{ph}}}}{t} .
$$

The time evolution of $n(t)$ is widely independent of the width $\Gamma$ of the initial distribution function at $t=0$ as one can see comparing the lowest curves in Figs. 2 and 3.

\section{Low-temperature electron-phonon relaxation in clean metals}

The low-frequency Eliashberg function is quadratic as a function of frequency, $\alpha^{2} F(\omega)=\lambda\left(\omega / \omega_{D}\right)^{2}$ in clean crystalline metals, which makes an analytical expression for the Fourier transform of Boltzmann equation (31) unavailable in terms of elementary functions. However we can approximate all relevant integrals numerically as

$$
\begin{gathered}
\int_{0}^{\infty} d \omega \frac{\omega^{2}}{\sinh (\omega / 2) \cosh (\omega / 2)} \approx 8.414, \\
\int_{0}^{\infty} d \omega \frac{\omega^{2} \sinh ^{2}(\xi / 2) \tanh (\omega / 2)}{\cosh \left(\frac{\omega+\xi}{2}\right) \cosh \left(\frac{\omega-\xi}{2}\right)} \approx \frac{3}{2} \xi^{2}+0.027 \xi^{4},
\end{gathered}
$$

and

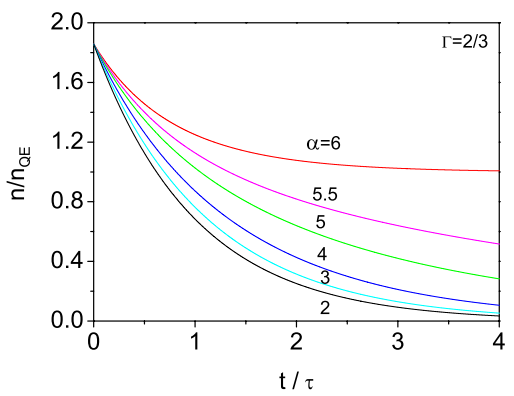

FIG. 3. (Color online) Relaxation of the total number of nonequilibrium excitations, $n$, for different e-e and e-ph scattering times characterized by parameter $\alpha$ [Eq. (51)] for the width $\Gamma$ $=2 / 3$ of the initial nonequilibrium distribution.

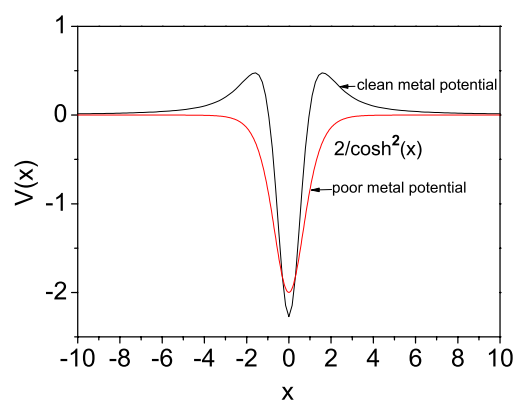

FIG. 4. (Color online) The effective potential energy of the Schrödinger counterpart of the Boltzmann equation with the e-ph collision integral in clean metals compared with the potential in poor metals.

$$
\int_{0}^{\infty} d \omega \frac{\omega^{2} \cos (\omega x / \pi)}{\sinh (\omega / 2)} \approx \frac{3 \pi^{2}}{2} V(x)
$$

where $V(x)$ is shown in Fig. 4. Then the corresponding Schrödinger-type equation for the Fourier transform of $\chi(\xi, t)$ becomes

$$
\tau_{\mathrm{ph}}^{\mathrm{cl}} \dot{\psi}(x, t)=\left[\frac{\partial^{2}}{\partial x^{2}}+0.178 \frac{\partial^{4}}{\partial x^{4}}+V(x)-0.568\right] \psi(x, t),
$$

where now

$$
\tau_{\mathrm{ph}}^{\mathrm{cl}}=\frac{\hbar^{3} \omega_{D}^{2}}{3 \pi^{3} \lambda\left(k_{B} T\right)^{3}} .
$$

The effective "potential" energy differs only marginally from the poor-metal case (Fig. 4). At large $t$ corresponding to large $x$ in Eq. (46), the fourth derivative of the low-energy extended eigenstates is small. Hence the asymptotic behavior of response functions in clean metals is qualitatively about the same as in poor metals,

$$
n(t) \propto \frac{\exp \left(-0.568 t / \tau_{\mathrm{ph}}^{\mathrm{cl}}\right)}{t},
$$

but the temperature dependence of the e-ph relaxation time is more pronounced, $\tau_{\mathrm{ph}}^{\mathrm{cl}} \propto 1 / T^{3}$. In principle, the clean-metal potential (Fig. 4) could have "resonances," states that are in the continuum but take a long time to leak out, resulting in some quantitative differences with the poor-metal relaxation.

\section{LOW-TEMPERATURE ELECTRON-PHONON RELAXATION COMBINED WITH ELECTRON- ELECTRON RELAXATION}

Finally let us combine both collision integrals into one Boltzmann equation. Performing its Fourier transformation as described in Secs. II and III yields the following Schrödinger-type equation in the poor-metal case:

$$
\tau \dot{\psi}(x, t)=\left[\frac{\partial^{2}}{\partial x^{2}}+\frac{\alpha}{\cosh ^{2}(x)}-1\right] \psi(x, t),
$$

where 


$$
\tau=\frac{\tau_{\mathrm{e}} \tau_{\mathrm{ph}}}{\tau_{\mathrm{e}}+\tau_{\mathrm{ph}}}
$$

and

$$
\alpha=\frac{2 \tau_{\mathrm{e}}+6 \tau_{\mathrm{ph}}}{\tau_{\mathrm{e}}+\tau_{\mathrm{ph}}} .
$$

There are two bound states of the corresponding steadystate Schrödinger-type equation

$$
\left[\frac{\partial^{2}}{\partial x^{2}}+\frac{\alpha}{\cosh ^{2}(x)}\right] \psi(x)=-E \psi(x)
$$

because $\alpha$ is larger than 2 but smaller than $6 .^{28}$ The excited odd state has the eigenfunction

$$
\psi_{1}(x) \propto \frac{\tanh (x)}{[\cosh (x)]^{\left|E_{1}\right|^{1 / 2}}}
$$

and the energy

$$
E_{1}=-\frac{1}{4}\left[(1+4 \alpha)^{1 / 2}-3\right]^{2},
$$

which determines the asymptotic behavior of all linear response functions. In particular the number of excitations decays at large $t$ as

$$
n(t) \propto \exp \left[-\frac{\left(1+E_{1}\right) t}{\tau}\right] .
$$

When both relaxations are involved, the time evolution of $n(t)$ calculated using Eq. (49) with different initial distributions differs qualitatively from TTM relaxation as shown in Figs. 2 and 3. In fact electrons cannot attain the thermal quasiequilibrium at any $\alpha$ less than 6, in agreement with the numerical results of Ref. 26. Moreover the exact relaxation rate $\gamma=1+E_{1}$ depends on the ratio of the electron-electron relaxation time [Eq. (12)] and the electron-phonon relaxation time [Eq. (41)],

$$
r=\frac{\tau_{\mathrm{e}}}{\tau_{\mathrm{ph}}} \approx \frac{2 \lambda}{\pi \mu_{C}^{2}} \frac{E_{F}}{\hbar \omega_{D}} .
$$

Using Eq. (55), we find

$$
\gamma=c(r) \frac{\pi^{3} \lambda\left(k_{B} T\right)^{2}}{2 \hbar^{2} \omega_{D}},
$$

where

$$
c(r)=\frac{3 \sqrt{(1+r)(25+9 r)}-7 r-15}{2 r} .
$$

This coefficient changes from $c(r)=1$ at $r=\infty$ up to $c(r)$ $=8 / 5$ at $r=0$.

The TTM relaxation rate $\gamma_{\text {Tlow }}$ at low temperatures is readily obtained with the Eliashberg function $\alpha^{2} F(\omega)$ $=\lambda \omega / 2 \omega_{D}$ using Eqs. (4) and (10) of Refs. 14 and 33. Linearizing Eq. (10) of Ref. 14 with respect to the temperature difference $T_{e}(t)-T \ll T$ yields

$$
\gamma_{T \text { low }}=\frac{4 \pi^{3} \lambda\left(k_{B} T\right)^{2}}{5 \hbar^{2} \omega_{D}} .
$$

The ratio of our exact relaxation rate to the TTM rate is

$$
\frac{\gamma}{\gamma_{\text {Tlow }}}=\frac{5}{8} c(r) \text {. }
$$

If e-e collisions are much faster than e-ph collisions $(r \rightarrow 0)$, this ratio is 1 , justifying the TTM approximation also at low temperatures in the limit $t \rightarrow \infty, r \rightarrow 0$. However at low temperatures $r$ is not necessarily small as assumed in TTM even at small $\lambda \ll \mu_{C}$ because the Fermi energy in Eq. (56) is often much larger than the phonon energy. Just the opposite limit $r \rightarrow \infty$ is feasible at a sizable $\lambda$. In this limit the exact relaxation rate is slower than the low-temperature TTM rate, $\gamma / \gamma_{\text {Tlow }}=5 / 8$, so that one may underestimate the electronphonon coupling constant by about two times using TTM. Also an illegitimate fitting of experimental rates measured at temperatures below $\hbar \omega_{D} / k_{B}$ with the theoretical hightemperature TTM rate $\gamma_{T}$ (Ref. 14) (see Sec. III A) may underestimate $\lambda$ by about $\left(\hbar \omega_{D} / \pi k_{B} T\right)^{3}$ times in poor metals and much more in clean metals.

\section{CONCLUSIONS}

In conclusion, using the auxiliary Fourier transform, we have mapped the linearized Boltzmann equation with the electron-electron collision integral onto a Schrödinger-type equation with imaginary time allowing for a simple analytical solution. A similar mapping is also found for the electronphonon collision integral at low temperatures in both poor and clean metals. We have analytically traced the time and energy evolution of the nonequilibrium electron distribution function on any time scale and found its asymptotic relaxation rate at $t \rightarrow \infty$.

A low-temperature relaxation rate strongly depends on the temperature: $\gamma \propto T^{2}$ and $\gamma \propto T^{3}$ in poor and clean metals, respectively. The Pauli exclusion principle slows down e-e relaxation, so that e-e and e-ph collisions are strongly entangled at low temperatures. We have shown that an electron gas cannot attain a thermal quasiequilibrium distribution by e-e collisions (Figs. 2 and 3), in agreement with earlier numerical integrations of the Boltzmann equation. ${ }^{26}$ The rate of return to the equilibrium is not governed solely by electronphonon processes, but also involves the electron-electron relaxation time, $\gamma=c(r) \gamma_{\text {Tlow }}$, via the coefficient $c(r)$, which depends on the ratio $r$ of the e-e collision time to the e-ph collision time. The exact relaxation rate $\gamma$ recovers its quasiequilibrium TTM value $\gamma=\gamma_{\text {Tlow }}$ only in the limit of the negligible e-ph coupling, $\lambda \ll \pi \mu_{C}^{2} \hbar \omega_{D} / 2 E_{F} \lesssim 0.01$. In poor metals the physically realistic ratio $r$ is large at low temperatures and the exact relaxation rate is slower than the TTM rate, $\gamma=5 \gamma_{\text {Tlow }} / 8$.

At high temperatures, $T \gg \hbar \omega_{D} / k_{B}$, we have reduced the e-ph collision integral to the differential Landau-FokkerPlanck form. Using this form we have shown that the deviation of the electron distribution from quasiequilibrium population might not have much influence on the energy relaxation, so that TTM (Refs. 13 and 14) is a reliable ap- 
proximation at high temperatures, if the e-e collisions are fast enough.

Our theory opens up a perspective of determinations of both important microscopic parameters $\lambda$ and $\mu_{C}$ using single-parameter $(\alpha)$ fitting of response functions in pumpprobe experiments at low temperatures (Figs. 2 and 3). It also allows for an analytical approach to the integral Boltzmann equation for the case of a steady-state source of excitations as in a current-carrying state. In the latter case relaxation times could be different because the current-carrying state does not have a distribution function that depends only on energy, as assumed here. The theory could be further extended beyond the assumption that phonons remain in equilibrium by including a linearized Boltzmann equation for the nonequilibrium phonon distribution function.

\section{ACKNOWLEDGMENTS}

We are grateful to Alexander Veselov and Rajmund Krivec for illuminating discussions of the Calogero-MoserSutherland equations and to Dragan Mihailovic for sharing with us his insight into nonequilibrium phenomena in metals and high-temperature superconductors. The work was supported by the Slovenian Research Agency (ARRS) (Grant No. 430-66/2007-17) and by EPSRC-GB (Grant No. EP/ D035589/1).
${ }^{1}$ S. D. Brorson, A. Kazeroonian, J. S. Moodera, D. W. Face, T. K. Cheng, E. P. Ippen, M. S. Dresselhaus, and G. Dresselhaus, Phys. Rev. Lett. 64, 2172 (1990).

${ }^{2}$ R. W. Schoenlein, W. Z. Lin, J. G. Fujimoto, and G. L. Eesley, Phys. Rev. Lett. 58, 1680 (1987).

${ }^{3}$ H. E. Elsayed-Ali, T. B. Norris, M. A. Pessot, and G. A. Mourou, Phys. Rev. Lett. 58, 1212 (1987)

${ }^{4}$ R. H. M. Groeneveld, R. Sprik, and A. Lagendijk, Phys. Rev. Lett. 64, 784 (1990).

${ }^{5}$ S. D. Brorson, J. G. Fujimoto, and E. P. Ippen, Phys. Rev. Lett. 59, 1962 (1987).

${ }^{6}$ G. L. Eesley, J. Heremans, M. S. Meyer, G. L. Doll, and S. H. Liou, Phys. Rev. Lett. 65, 3445 (1990).

${ }^{7}$ S. G. Han, Z. V. Vardeny, K. S. Wong, O. G. Symko, and G. Koren, Phys. Rev. Lett. 65, 2708 (1990).

${ }^{8}$ S. V. Chekalin, V. M. Farztdinov, V. V. Golovlyov, V. S. Letokhov, Yu. E. Lozovik, Yu. A. Matveets, and A. G. Stepanov, Phys. Rev. Lett. 67, 3860 (1991).

${ }^{9}$ W. Albrecht, Th. Kruse, and H. Kurz, Phys. Rev. Lett. 69, 1451 (1992).

${ }^{10}$ C. J. Stevens, D. Smith, C. Chen, J. F. Ryan, B. Podobnik, D. Mihailovic, G. A. Wagner, and J. E. Evetts, Phys. Rev. Lett. 78, 2212 (1997)

${ }^{11}$ J. Demsar, B. Podobnik, V. V. Kabanov, T. Wolf, and D. Mihailovic, Phys. Rev. Lett. 82, 4918 (1999).

${ }^{12}$ V. V. Kabanov, J. Demsar, B. Podobnik, and D. Mihailovic, Phys. Rev. B 59, 1497 (1999).

${ }^{13}$ M. I. Kaganov, I. M. Lifshits, and L. B. Tanatarov, Zh. Eksp. Teor. Fiz. 31, 232 (1956) [Sov. Phys. JETP 4, 173 (1957)].

${ }^{14}$ P. B. Allen, Phys. Rev. Lett. 59, 1460 (1987).

${ }^{15}$ G. P. Segre, N. Gedik, J. Orenstein, D. A. Bonn, R. Liang, and W. N. Hardy, Phys. Rev. Lett. 88, 137001 (2002); N. Gedik, P. Blake, R. C. Spitzer, J. Orenstein, R. Liang, D. A. Bonn, and W. N. Hardy, Phys. Rev. B 70, 014504 (2004).
${ }^{16}$ A. Rothwarf and B. N. Taylor, Phys. Rev. Lett. 19, 27 (1967).

${ }^{17}$ J. Demsar, R. D. Averitt, V. V. Kabanov, and D. Mihailovic, Phys. Rev. Lett. 91, 169701 (2003).

${ }^{18}$ V. V. Kabanov, J. Demsar, and D. Mihailovic, Phys. Rev. Lett. 95, 147002 (2005).

${ }^{19}$ R. A. Kaindl, M. A. Carnahan, D. S. Chemla, S. Oh, and J. N. Eckstein, Phys. Rev. B 72, 060510(R) (2005).

${ }^{20}$ L. Perfetti, P. A. Loukakos, M. Lisowski, U. Bovensiepen, H. Eisaki, and M. Wolf, Phys. Rev. Lett. 99, 197001 (2007).

${ }^{21}$ J. X. Zhu, E. E. M. Chia, T. Tamegai, H. Eisaki, K. H. Oh, S. I. Lee, and A. J. Taylor, arXiv:0806.2664 (unpublished).

${ }^{22}$ A. S. Alexandrov and N. F. Mott, Rep. Prog. Phys. 57, 1197 (1994), and references therein.

${ }^{23}$ P. W. Anderson, Science 316, 1705 (2007), and references therein.

${ }^{24}$ J. Demsar, R. D. Averitt, K. H. Ahn, M. J. Graf, S. A. Trugman, V. V. Kabanov, J. L. Sarrao, and A. J. Taylor, Phys. Rev. Lett. 91, 027401 (2003).

${ }^{25}$ K. H. Ahn, M. J. Graf, S. A. Trugman, J. Demsar, R. D. Averitt, J. L. Sarrao, and A. J. Taylor, Phys. Rev. B 69, 045114 (2004).

${ }^{26}$ R. H. M. Groeneveld, R. Sprik, and Ad. Lagendijk, Phys. Rev. B 51, 11433 (1995).

${ }^{27}$ V. I. Karas and I. F. Potapenko, Zh. Vychisl. Mat. Mat. Fiz. 46, 307 (2006) [Comput. Math. Math. Phys. 46, 294 (2006)].

${ }^{28}$ L. D. Landau and E. M. Lifshitz, Quantum Mechanics (Pergamon, Oxford, 1977).

${ }^{29}$ We thank A. P. Veselov for a hint which led us to this expression.

${ }^{30}$ D. Belitz, Phys. Rev. B 36, 2513 (1987).

${ }^{31}$ D. Belitz and M. N. Wybourne, Phys. Rev. B 51, 689 (1995).

${ }^{32}$ B. I. Belevtsev, Yu. F. Komnik, and E. Yu. Beliayev, Phys. Rev. B 58, 8079 (1998).

${ }^{33}$ We noticed that a multiplier $\pi^{2} N_{c} N\left(E_{F}\right) / 3$ is missing in the second term of Eq. (4) of Ref. 14. 